\title{
Simple Synthesis of Ag Nanoparticles Arranged in 1D and 2D Grating- like Structures
}

\author{
Giorgio Baraldi, Stephanie Reynaud, Anthony Cazier, Yaya Lefkir, Nicolas Crespo-Monteiro, Francis Vocanson, Nathalie \\ Destouches* \\ Univ Lyon, UJM Saint- Etienne, CNRS, Institut d'Optique Graduate School, Laboratoire Hubert \\ Curien UMR 5516, F-42043, SAINT-ETIENNE, France \\ *Email: nathalie.destouches@univ-st-etienne.fr
}

\begin{abstract}
Laser-induced self-organization of matter is a guarantee of efficient and cost-effective processes. Ultra-fast laser has been generally used to induce organization of metallic nanoparticles, which opened new ways to the fabrication of plasmonic nanostructures with tailored morphological and, thus, optical properties. Our group have shown that self-organization of nanoparticles could be also obtained in the case of thin films loaded with metallic ions using CW lasers. In this work, we use illumination with CW laser to induce the formation of a self-organization resembling a two dimensional (2D) gratinglike structure. With the help of an ArKr laser, a solid state laser and two half-wave plates used to rotate the laser linear polarization, we first demonstrate our capability of producing one dimensional (1D) gratings. Then, we study the effect of exposing a same sample area to two laser beams, simultaneously, with perpendicular polarization orientations. Scanning electron microscopy (SEM) is used to study the morphology of samples after laser exposure.
\end{abstract}

DOI: $10.2961 /$ jlmn.2016.03.0005

Keywords: silver, nanoparticles, thin film, $\mathrm{TiO}_{2}$, laser, self-organization, 1D grating, 2D grating

\section{Introduction}

Noble metal nanoparticles found applications in many fields because of their optical properties mainly determined by the electromagnetic excitation of the localized surface plasmon resonance (LSPR) [1]. In the case of nanoparticle arrays, the electromagnetic interaction between neighboring nanoparticles can influence the optical response of the whole assembly. In particular, the electromagnetic interaction between neighboring nanoparticles, mediated by nearor far-field coupling effects, can lead to the appearance of collective modes like Fano-resonances [2], and light waveguiding [3] which are of great interest for application of nanoparticle in optical nanocircuits [4], solar cells [5,6] and security technologies [7]. In recent years, though, many efforts have been addressed to explore different large-scale fabrication techniques to improve the control over organization of nanoparticles in one (1D) and two dimensional (2D) patterns to discover new optical properties [1]. Besides very precise, but also time-expensive, top-down fabrication techniques, like electron beam lithography, bottomup methods based on physical methods can be employed to induce $1 \mathrm{D}$ and 2D self-organization of metal nanoparticles. For example, facetted as well as rippled dielectric and semiconductor surfaces have been successfully used to induce the deposition of $1 \mathrm{D}$ assembly of nanoparticles [8,9], while anodically grown $\mathrm{TiO}_{2}$ nanotubes and pre-patterned polymer surfaces have been used to obtain higher degree of organization, i.e. 2D [10]. In all these cases, though, specific arrangement of metal nanoparticles is obtained using patterned substrate, whose preparation is not necessarily straightforward. A different approach, which is compatible with unpatterned substrates, is based on using laser light to directly induce nanoparticle organization. Laser interference as well as the use of amplitude or phase masks have been successfully used to produce $1 \mathrm{D}$ and 2D patterns of metal nanoparticles supported or embedded on solid sub- strates [11,12]. Nonetheless, nanoparticle organization can be also obtained without tailoring the characteristics of the incident beam. Kampfe et al. [13] and Kiesow et al. [14], for example, used ultrashort laser pulses to induce the formation of periodic line patterns of metal nanoparticles embedded in polymer films. Later on, Eurenius et al. [15], which observed that Au and Ag nanoparticles deposited by electron beam and thermal evaporation, respectively, on a $\mathrm{Si}_{3} \mathrm{~N}_{4}$ membrane could be organized in $1 \mathrm{D}$ and $2 \mathrm{D}$ patterns by illuminating them using a single ns laser pulse working at a wavelength able to excite the LSPR of nanoparticles. Similar results have been reported by Loeschner et al. [16]. They observed 1D and 2D grating-like nanostructures when irradiating thin polymer films containing metal (Ag, $\mathrm{Au}, \mathrm{Cu}$ ) nanoparticles, deposited by thermal evaporation, using fs laser pulses. In these works, grating-like nanostructures result from a periodic modification of nanoparticle shape indirectly induced by the distribution of the electric field inside the studied films. Goutaland et al. [17] introduced a different approach based on UV continuouswave (CW) laser illumination of soda lime glass doped with Ag ions to induce the formation of periodic aligned nanoparticles. In this case, silver diffusion processes along with the formation of periodic surface structures, induced by light intensity distribution in the film, are responsible for the observed nanoparticle formation and organization. Nevertheless, the area containing periodic patterns is quite limited and only located at the vertical poles of laser spot. Following a similar approach, our group have shown that self-organization of nanoparticles could be also obtained in the case of thin films loaded with ions and small metallic nanoparticles when exciting the latter at resonance [18]. In particular, we have already demonstrated that $1 \mathrm{D}$ gratinglike structure consisting of metal nanoparticles can be obtained when an amorphous titania $\left(\mathrm{TiO}_{2}\right)$ thin film loaded with Ag salt, deposited on glass substrate, is illuminated 
with visible CW laser. Such periodic structures can be produced with different wavelengths lying in the plasmon resonance of Ag nanoparticles, their period is a fraction of the laser wavelength and their orientation parallel to the laser polarization. In addition, the long-order of these periodic nanostructures was also reported. This type of organized $\mathrm{Ag}: \mathrm{TiO}_{2}$ nanocomposite is important since it can be used for security [20] and plasmonic photocatalysis [21] applications.

In this work, we use a CW light illumination to induce the formation of self-organized plasmonic nanostructures in one dimensional (1D) grating-like structures and then we explore the possibility to induce two dimensional (2D) organization. In particular, we propose a simple synthesis method to achieve this result based on simultaneous illumination with two laser beams with crossed polarization. The obtained results are interpreted in terms of the models previously developed within our group based on the mechanisms of oxidation and reduction of Ag ions [19] and on the optical interference between the incident field and the light scattered by nanoparticles within the film [18].

\section{Experimental setup}

We performed experiments on a $230 \pm 50 \mathrm{~nm}$ thick $\mathrm{TiO}_{2}$ loaded with silver salt, deposited on glass substrates by dipcoating. To this purpose, we follow a standard procedure reported in our previous work [22]. After deposition the film is amorphous and mesoporous with pores with typical size ranging from $5 \mathrm{~nm}$ to $20 \mathrm{~nm}$. Silver is introduced in the $\mathrm{TiO}_{2}$ matrix in ionic state by impregnating the film in an aqueous ammoniacal silver nitrate solution $(1.5 \mathrm{M})$ for 30 minutes. Samples are then rinsed with pure water, dried 12 hours in dark at room temperature and exposed to UV light (400 $\mu \mathrm{W} \cdot \mathrm{cm}^{-2}$ at $254 \mathrm{~nm}$ wavelength) for 30 minutes. As a result of photocatalytic behaviour of $\mathrm{TiO}_{2}$, silver nanoparticles with average size of $3 \mathrm{~nm}$ are formed. UV exposure is needed to make the film more sensitive to laser exposure $[18,19]$.

Laser exposures are performed using an $\mathrm{ArKr}$ and solid state CW lasers working at $488 \mathrm{~nm}$ and $532 \mathrm{~nm}$, respectively. The beam shape is slightly different in the two cases. For the $\mathrm{ArKr}$ it is circular while for solid state laser it is rather elliptical. Nonetheless in the two cases the intensity distribution can be described by a Gaussian function. The laser beam arrives on the sample surface under normal incidence and it is focussed with a $10 \times$ microscope objective (Olympus MPlan N, N.A. 0.25). In this configuration, we obtain a spot at the focal plane with typical size of around $10 \mu \mathrm{m}$ and $8 \mu \mathrm{m}$ for $488 \mathrm{~nm}$ and $532 \mathrm{~nm}$ lasers, respectively. Focusing is needed to reach the power intensity threshold required to induce growth of Ag nanoparticles within the film. A half-wave plate is inserted in the optical path of the laser beam to allow rotating its linear polarization and a shutter is used to control the passage of laser beam. We consider two configurations. In the first one the laser beam at $488 \mathrm{~nm}$ is divided into two beams, the polarization of one of these beams is rotated by $90^{\circ}$ before recombining both beams with a second beam splitter in the same direction. The sample is then illuminated with two parallel and perfectly overlapping beams that do not interfere because they have cross polarizations. In the second case, the con- figuration is the same except the fact that two different wavelengths are used for cross polarizations.

For laser exposure, samples are mounted on motorized translational stages that can move at constant speed laterally and vertically. Lateral movements allow performing laser illumination on a specific sample area, while vertical movements are used to change/adjust laser focusing. A software that controls and synchronizes the stage movements with the shutter opening and closing is used to automatically perform laser exposure on large areas.

Sample morphology before and after laser exposure is studied using scanning (SEM) electron microscopy. The used SEM is a FEI NovananoSEM 200 model, working in low vacuum mode equipped with a Helix detector. Under this condition, microscopy could be performed on our composite films and embedded Ag nanoparticles could be imaged.

\section{Results}

We first study our capability to properly produce 1D nanoparticle gratings with lines oriented in perpendicular directions. In this sense, it is important to remember that self-organization of Ag nanoparticles can be achieved only when exposure conditions are properly optimized. Once the laser focus and power are fixed, a well-determined scan speed threshold must be overcome to observe nanoparticle growth and organization. To this purpose, we have performed several tests and we found that for ArKr laser working at $488 \mathrm{~nm}$, the best values were in the range from 200 $\mathrm{mW}$ to $250 \mathrm{~mW}$ for the laser power (on the sample surface), and from $250 \mu \mathrm{m} \cdot \mathrm{s}^{-1}$ to $300 \mu \mathrm{m} \cdot \mathrm{s}^{-1}$ for the scan speed. In addition the laser was slightly defocussed.

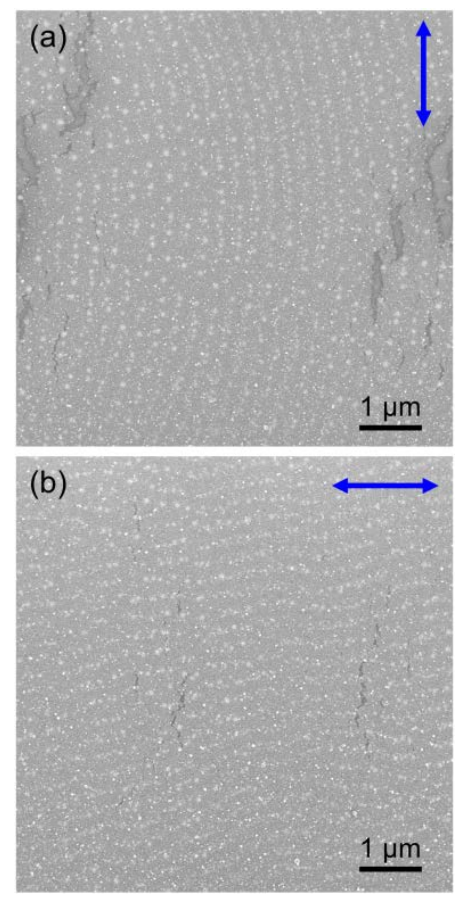

Fig. 1 Plan-view SEM micrographs of films illuminated with single laser scan at $488 \mathrm{~nm}$ with polarization oriented (a) vertically and (b) horizontally. Blue arrows indicate the orientation of the incident electric field.

Fig. 1 shows plan-view SEM micrographs of two different areas of a sample illuminated under the mentioned 
conditions by single laser scan with laser linear polarization oriented vertically and horizontally. Scans are performed from right to left. In the micrographs $\mathrm{Ag}$ corresponds to elements showing bright contrast, while the grey background corresponds to the $\mathrm{TiO}_{2}$ host matrix. In both cases, we observe that lines containing Ag nanoparticles are formed after laser exposure. Their period is, approximately, $280 \mathrm{~nm}$ and their orientation is parallel to the laser polarization (see arrows in Figs. 1a and b). The nanoparticle size distribution is quite broad, ranging from $20 \mathrm{~nm}$ to $80 \mathrm{~nm}$, in (a) and (b). These values were determined on a SEM micrograph at higher magnification (not shown) using an image processing procedure developed within our group. SEM micrographs also reveal that several cracks are formed in the film and that grating lines are not perfectly straight.

We have already reported that the period of the nanoparticle gratings depends on the laser wavelength. Fig. 2 shows the plan-view SEM micrograph of a film illuminated with the solid state laser working at $532 \mathrm{~nm}$. In this case the laser polarization was rotated to be horizontal. The exposure conditions were similar to the ones used in the previous case, with the difference that the power was fixed at $300 \mathrm{~mW}$.

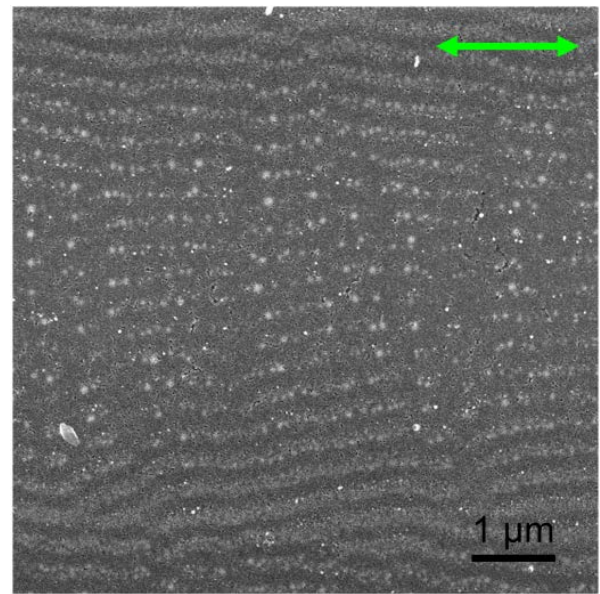

Fig. 2 Plan-view SEM micrograph of film illuminated using the solid state laser working at $532 \mathrm{~nm}$. Green arrow indicates the orientation of the incident electric field.

Similarly to the previous case (Fig. 1), we observe that Ag nanoparticles are formed after laser exposure and that they are confined in horizontal lines parallel to the laser polarization (see black arrow). The period of the grating is $330 \mathrm{~nm}$. We observe at the bottom and top of the micrograph (at the edge of the laser scan line) that smaller nanoparticles are formed with respect to the centre of the line. In general, compared to the case of illumination with $488 \mathrm{~nm}$, nanoparticles are smaller in this case. Finally, an important difference with respect to the illumination at $488 \mathrm{~nm}$ is that no cracks in the film are observed.

Fig. 3 shows plan-view SEM micrographs of a sample illuminated using simultaneous laser exposures with two beams having crossed polarizations. (a) corresponds to the exposure to $488 \mathrm{~nm}$ and (b) to the overlapping of the 488 $\mathrm{nm}$ and $532 \mathrm{~nm}$ beams. The exposure conditions were the ones used for exposure with one beam, apart from the power whose value was halved since in this case we have to take into account that the power arriving on the sample surface is the sum of the two beams.

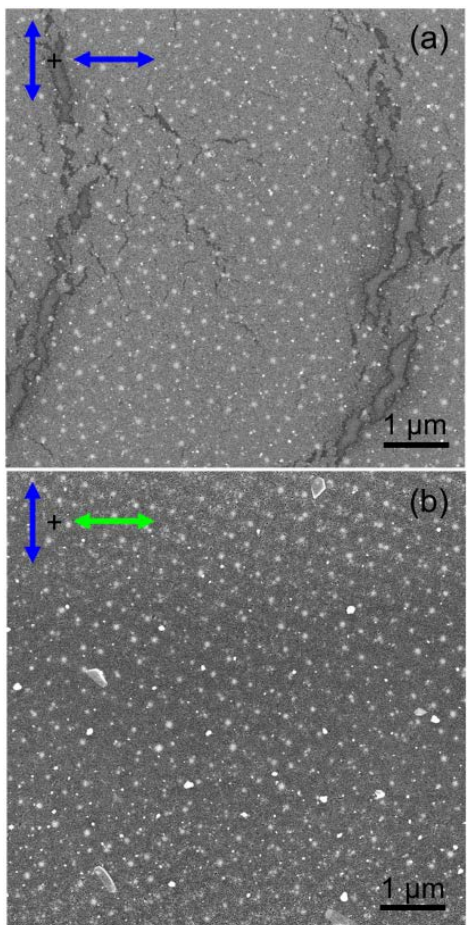

Fig. 3 Plan-view SEM micrograph of films illuminated using simultaneous exposure using (a) the $488 \mathrm{~nm}$ beam separated into two using a beam splitter and (b) overlapping the $488 \mathrm{~nm}$ and 532 $\mathrm{nm}$ beams. Blue and green arrows indicate the orientation of the incident electric field for $488 \mathrm{~nm}$ and $532 \mathrm{~nm}$ wavelengths, respectively.

We observe that the morphology of the nanostructures is different from previous cases where a single beam was used (Figs. 1 and 2). Nanoparticles are not formed in 1D gratings but they are rather homogeneously distributed in two dimensions in both (a) and (b). The average distance between nanoparticles is around $280 \mathrm{~nm}$ in all directions in (a), while in (b) it is $280 \mathrm{~nm}$ along the horizontal axis of the figure and, approximately, $330 \mathrm{~nm}$ along the vertical. In addition, the nanoparticle size distribution is narrower than for illumination using a single laser beam, and it ranges from $50 \mathrm{~nm}$ to $70 \mathrm{~nm}$. The two SEM micrographs also reveal that in (b) the film surface in free of cracks, while it is not the case for (a).

\section{Discussion}

The growth and organization of Ag nanoparticles in 1D gratings under CW illumination, as observed in Figs. 1 and 2 , can be explained in terms of two main mechanisms. The first relates to the plasmon-induced temperature increase that promotes $\mathrm{Ag}$ ion reduction and growth of small $\mathrm{Ag}$ nanoparticles initially formed in the film [19]. The second relates to the interference between the incoming laser beam and the light scattered by nanoparticles trapped in the guided modes of the $\mathrm{TiO}_{2}$, which is responsible for inducing the growth of nanoparticles in a periodic pattern [18]. We can thus associate the different periods observed in Fig. 1 and Fig. 2 to the fact that two different laser wavelengths promote a different modulation of the field inside the film. In particular, as reported earlier [18], the shortest the wavelength, the smaller the period. In addition, the lines of the 
grating are always oriented parallel to the laser polarization (Figs. 1 and 2) since laser beam is mainly exciting the dipolar mode of the LSPR, which mainly scatters light in the plane perpendicular to the incident polarization. Finally, the cracks observed in the film in Fig. 1 can be associated to an excessive heating of the $\mathrm{TiO}_{2}$ matrix resulting from the excitation of the LSPR. This is likely to be related to the fact that the initially formed nanoparticles exhibit higher light absorption in the blue rather than in the green wavelength range because of their reduced size $(\sim 3 \mathrm{~nm})$.

The homogeneous organization of nanoparticles in two dimensions observed in Fig. 3 is quite unusual, but it can be explained in terms of the already mentioned mechanisms, since in (a) the distance between nanoparticles resembles that of the nanoparticle gratings in Fig. 1 in all directions, and in (b) it is similar to those observe for grating formed at $488 \mathrm{~nm}$ and $532 \mathrm{~nm}$ along the horizontal and vertical directions, respectively. The fact that in these cases nanoparticles are more homogeneously distributed within the film surface can be related to the fact that two plane waves with crossed polarization arrive simultaneously on the sample surface. As a result, they can give rise to a more complex interference pattern responsible for the observed nanoparticle organization. This resulting interference pattern can also be responsible for the observed narrowing of nanoparticle size distribution with respect to the case of single laser beam as a result of a more isotropic distribution of the modulated electromagnetic field within the film. Finally, the presence of the cracks in (a) further remarks the effect of temperature increase within the film induced by the excitation of the LSPR also in this case, and the importance to be able to control it to prevent $\mathrm{TiO}_{2}$ damaging.

\section{Conclusions}

In this work we reported that self-organization of metal nanoparticles in 1D gratings with different orientations can be obtained by simply rotating the linear polarization of the laser. Following an approach based on simultaneous illumination of the $\mathrm{Ag}: \mathrm{TiO}_{2}$ film with two laser beams, of the same or different wavelengths, with crossed polarizations we have also shown that nanoparticles can be organized in $2 \mathrm{D}$, in the sense that the formed nanoparticles are separated by a similar average distance in two directions. The value of the distance mirrors the one observed for $1 \mathrm{D}$ gratings hinting at the fact that the mechanisms leading to the formation of nanoparticles are, basically, the same, while the different spatial organization can be related to the modulation of the field within the film resulting from the interaction between the laser beam and the light scattered by nanoparticles in two perpendicular directions. SEM micrographs also evidenced the presence of cracks in the film when using shorter laser wavelengths. This remarks that the plasmon-induced temperature increase is more pronounced when illuminating at $488 \mathrm{~nm}$ than at $532 \mathrm{~nm}$, probably due to the initially formed nanoparticles that absorb more efficiently in the blue because of their reduced size.

Further experiments are under study to transfer this technique to large areas and to improve the pattern homogeneity.

\section{Acknowledgments}

This work has been supported by ANR in the framework of project PHOTOFLEX 12-NANO-0006. GB acknowledges the financial support in the framework of the "Programme Avenir Lyon Saint-Etienne" of the Université de Lyon (ANR-11-IDEX-0007), within the Program "Investissements d'Avenir" operated by the French National Research Agency. The research leading to these results has received funding from the People Program (Marie Curie Actions) of the European Union's Seventh Framework Programme (FP7/2007-2013) under REA grant agreement $n^{\circ}$ PCOFUND-GA-2013-609102, through the PRESTIGE programme coordinated by Campus France.

\section{References}

[1] Z. Nie, A. Petukhova, and E. Kumacheva: Nature Nanotechnology, 5, (2010), 15

[2] S. Bakhti, N. Destouches, A. V. Tishchenko: Book Chapter Reviews in Plasmonics (2015), pp 19

[3] N. Dmitry, A. V. Lavrinenko, C. M. Sotomayor Torres: Optics Express, 12, (2004), 617

[4] J. S. Huang, T. Feichtner, P. Biagioni, B. Hecht: Nano Lett., 9, (2009), 1897

[5] S. Pillai, K. R. Catchpole, T. Trupke, M. A. Green: J. Appl. Phys. 101, (2007), 093105

[6] V. E. Ferry, L. A. Sweatlock, D. Pacifici, H. A. Atwater: Nano Lett., 8, (2008), 4391

[7] R. Parker: J. Opt. A: Pure Appl. Opt., 2, (2000), 15

[8] E. Fort, C. Ricolleau, and J. Sau-Peyo: Nano Lett., 3, (2003), 65

[9] F. Cuccureddu, S. Murphy, I. V. Shvets, M. Porcu, and H. W. Zandbergen: Nano Lett., 8, (2008), 3248

[10] J. E. Yoo, K. Lee, M. Altomare, E. Selli, and P. Schmuki: Angew. Chem. Int. Ed., 52, (2013), 7514

[11] M. Mäder, T. Höche, J. W. Gerlach, S. Perlt, J. Dorfmüller, M. Saliba, R. Vogelgesang, K. Kern, and B. Rauschenbach: Nano Lett. 10, (2010), 47

[12]R. J. Pelaez, C. N. Afonso, J. Bulír, M. Novotny, J. Lančok, and K. Piksová: Nanotechnology, 24, (2013), 095301

[13] M. Kampfe, H. Graener, A. Kiesow, and A. Heilmann: Appl. Phys. Lett., 79, (2001), 1876

[14]A. Kiesow, S. Strohkark, K. Löescher, A. Heilmann, A. Podlipensky, A. Abdolvand, and G. Seifert: Appl. Phys. Lett. 86, (2005), 153111

[15]L. Eurenius, C. Hägglund, E. Olsson, B. Kasemo, and D. Chakarov: Nature Photonics, 2, (2008), 360

[16]K. Löescher, G. Seifert, and A. Heilmann: J. Appl. Phys., 108, (2010), 073114

[17]F. Goutaland, J.-P. Colombier, M. Cherif Sow, N. Ollier, and F. Vocanson: Optics Express, 21, (2013), 3178931799

[18]N. Destouches, N. Crespo-Monteiro, G. Vitrant, Y. Lefkir, S. Reynaud, T. Epicier, Y. Liu, F. Vocanson, and F. Pigeon: J. Mater. Chem. C, 2, (2014), 6256

[19]Z. Liu, N. Destouches, G. Vitrant, Y. Lefkir, T. Epicier, F. Vocanson, S. Bakhti, Y. Fang, B. Bandyopadhyay, and M. Ahmed: J. Phys. Chem. C, 119, (2015), 9496

[20] J. Martinez-García, M. Hébert, A. Trémeau, N. Crespo-Monteiro and N. Destouches, Color and Imaging Conference, 1, (2015), 116 
[21] X. Zhang, Y. L. Chen, R.-S. Liu and D. P. Tsai, Rep. Prog. Phys. , 76, (2013), 046401

[22] N. Crespo-Monteiro, N. Destouches, L. Bois, F. Chassagneux, S. Reynaud, T. Fournel: Adv. Mater., 22, (2010), 3166

(Received: May 24, 2016, Accepted: August 14, 2016) 